\title{
Les infections nosocomiales : que faut-il faire?
}

«Ả l'hôpital, on vide l'ambulance et on la désinfecte ensuite à fond. L'infirmière change ses vêtements extérieurs et on laisse toutes les couvertures à l'hôpital pour qu'elles soient lavées. [...] Outre cette désinfection à l'hôpital [...] les infirmières et les ambulanciers se lavent et se changent avant de partir. Il n'y a pas lieu de craindre, selon nous, que l'hôpital devienne un foyer d'infection ${ }^{1} . »$

$\mathrm{P}$ endant la majeure partie de leur histoire, les hôpitaux étaient avant tout un refuge pour les démunis et les mourants, un endroit que les riches évitaient. Au $\mathrm{XIX}^{\mathrm{e}}$ et au début du $\mathrm{XX}^{\mathrm{e}}$ siècles, ils servaient à traiter (et à contenir) des maladies infectieuses comme la variole (comme dans le cas que l'on vient de citer). Depuis une centaine d'années environ, et particulièrement vers la deuxième moitié du dernier siècle, les hôpitaux sont toutefois devenus une vitrine des miracles de la médecine et des technologies impressionnantes. Ce sont aussi des endroits dangereux, en partie à cause de la complexité du savoir médical - médicaments et interventions multiples, multiples spécialistes et multiples systèmes complexes de prestation de soins. On commet des erreurs. Les systèmes ont des défaillances².

Les demandes constantes de ressources afin de financer des technologies nouvelles et coûteuses taxent les budgets même des meilleurs administrateurs d'hôpital. Pour faire fonctionner de nouveaux appareils ou acheter plus de stents, ils doivent arracher de l'argent aux budgets de fonctionnement de base. Par ailleurs, ils ne peuvent en même temps négliger les services de base aux patients, ni les risques environnementaux auxquels les patients et le personnel sont exposés tous les jours. Les risques ne sont pas insignifiants.

Certains dangers nosocomiaux font l'objet d'une surveillance et d'un contrôle attentifs. Les membres du personnel qui travaillent avec des rayonnements portent des dosimètres qui captent constamment les rayonnements dans leur milieu de travail. Les systèmes d'alerte et de surveillance des doses de médicaments et des interactions deviennent la norme dans la plupart des établissements. On évalue actuellement d'autres systèmes sophistiqués à code à barres ou micropuce pour produits (et patients) afin de contrôler tout un éventail de dangers possibles.

La surveillance d'un éventail de plus en plus large d'agents microbiologiques de plus en plus résistants demeure toutefois moyenâgeuse. Les patients et les membres du personnel ne sont pas soumis à un dépistage de routine de ces agents. Les procédures de déclaration sont plutôt fragiles. Une fois que ces infections se sont déclarées, le confinement est improvisé et entravé par une infrastructure hospitalière qui, trop souvent, est dépassée de 100 ans. De nombreux patients entassés dans une seule pièce dotée d'une seule toilette et d'un seul lavabo (que le personnel utilise aussi) : voilà ce qu'on retrouve normalement dans la plupart des hôpitaux du Canada.

Le virus du SRAS était un nouvel organisme que nous n'é- tions pas prêts à affronter³. Les éclosions de Clostridium difficile, micro-organisme qu'il est possible de dépister au moyen d'un test très sensible et spécifique, n'auraient pas dû nous surprendre. Le système automatisé de production de rapports de laboratoire dans la plupart des hôpitaux aurait dû prévenir les équipes de lutte contre les infections et les administrateurs des hôpitaux. Les dirigeants de la santé publique auraient dû être prévenus. D'autres pays ont déclaré au cours des dernières années de telles éclosions dévastatrices de $C$. difficile. L'an dernier, un groupe d'experts du Royaume-Uni a publié un rapport détaillé sur la détection et le traitement de la diarrhée causée par $C$. difficile dans les hôpitaux, et a recommandé notamment que la déclaration de la présence de ce microorganisme soit obligatoire ${ }^{4}$.

«Un signal d'alerte», avons-nous déclaré après la crise du SRAS. Nous n'avons toutefois pas été sortis de notre sommeil. Les éclosions de $C$. difficile démontrent que le Canada a besoin d'un système national de surveillance des infections nosocomiales basé dans les hôpitaux, qui fonctionne en temps réel et auquel le public a accès par Internet, comme dans le cas de celui du Royaume-Uni ${ }^{5}$.

Il est urgent de moderniser les hôpitaux et d'en construire d'autres pour faire face à ces nouveaux risques environnementaux, mais il faudra du temps (et de l'argent). En attendant, il faudra aussi nous adapter. Dans le rapport que nous publions dans le présent numéro ${ }^{6}$ [voir page 466], le $\mathrm{D}^{\mathrm{r}}$ Pépin et ses collègues indiquent que la vancomycine est peut-être le médicament de premier choix pour traiter l'infection par $C$. difficile. À cause du taux de mortalité élevé lié à la diarrhée causée par C. difficile et du risque de créer des organismes résistants à la vancomycine, les Instituts de recherche en santé du Canada (IRSC) devraient envisager de débloquer des fonds d'urgence pour cette recherche. Afin de confiner ces éclosions continues de microorganismes résistants, nous avons besoin de nouveaux systèmes qui permettent de détecter des taux nouveaux ou inhabituels d'infection et qui fournissent des données de surveillance que l'on puisse diffuser rapidement à grande échelle. — $\mathcal{F A M C}$

\section{Références}

1. The new ambulance station of the Metropolitan Asylums Board. BM7 1881;ii:567.

2. Baker GR, Norton PG, Flintoft V, Blais R, Brown A, Cox J, et al. The Canadian Adverse Events Study: the incidence of adverse events among hospital patients in Canada. FAMC 2004;170(11):1678-86.

3. Leçons tirées du SRAS [éditorial]. FAMC 2003;168(11):1383.

4. National Clostridium difficile Standards Group. National Clostridium difficile Standards Group: report to the Department of Health. 7 Hosp Infect 2004;56 Suppl 1:1-38. Texte disponible à www.dh.gov.uk/assetRoot/04/06/76/51 /04067651.pdf (consulté le 3 août 2004).

5. Department of Health Mandatory Bacteraiemia Surveillance Scheme: NHS Trust, avril 2001-2004. www.dh.gov.uk/assetRoot/04/08/58/93/04085893.pdf (consulté le 28 juillet 2004).

6. Pépin J, Valiquette L, Alary ME, Villemure P, Pelletier A, Forget K, et al. Clostridium difficile-associated diarrhea in a region of Quebec from 1991 to 2003: a changing pattern of disease severity. 7AMC 2004;171(5):466-72. Article publié en accéléré le 4 août 2004 (DOI:10.1503/cmaj.1041104). 